\title{
Statine verbessern die antidepressive Wirkung
}

Fragestellung: Führt eine gleichzeitige Behandlung mit Statinen zu einer Verbesserung der antidepressiven Wirkung von selektiven Serotonin-Wiederaufnahme-Hemmern (SSRI)?

Hintergrund: Tierexperimentelle und erste randomisierte placebokontrollierte klinische Studien haben Hinweise auf eine verbesserte antidepressive (bzw. anxiolytische) Wirkung einer Kombinationstherapie von Fluoxetin mit einem Statin gegenüber einer alleinigen Behandlung mit Fluoxetin erbracht.

Patienten und Methodik: In Dänemark wurde eine landesweite naturalistische populationsbasierte Kohortenstudie mit allen in den Jahren von 1997 bis 2012 neu auf ein SSRI eingestellten Patienten durchgeführt, bei denen zu irgendeinem Zeitpunkt in diesem Intervall eine gleichzeitige Behandlung mit ei-

Köhler O, Gasse C, Petersen L et al. The Effect of Concomitant Treatment With SSRIs and Statins: A Population-Based Study. Am J Psychiatry 2016; 173: 807-15 kontakte wegen einer Depression, suizidales Verhalten und allgemeine Mortalität ausgewertet.

Ergebnisse: Es wurden 872.216 Patienten (Altersmedian 48 Jahre, $61,6 \%$ Frauen) mit Einstellung auf ein SSRI ermittelt, von denen 113.108 (13,0\%) gleichzeitig ein Statin erhielten. Die Kombinationsbehandlung führte im Vergleich mit alleiniger SSRI-Gabe zu einer signifikanten Risikoerniedrigung sowohl bezüglich psychiatrischer Klinikkontakte beliebiger Ursache (bereinigte Hazard Ratio [HR] 0,75; $95 \%$-Konfidenzintervall [KI] 0,69-0,82) als auch speziell wegen einer Depression (MDD) (bereinigte HR 0,64; $95 \%$-KI 0,55-0,75). Dabei war die gleichzeitige Gabe von SSRI und einem Statin im Vergleich zur SSRI-,,Monotherapie“ weder assoziiert mit einem Anstieg des allgemeinen Mortalitätsrisikos (bereinigte HR 1,04; 95\%-KI 0,96-1,12) noch mit vermehrtem suizidalem Verhalten (bereinigte HR 0,85; $95 \%$-KI 0,61 - 1,18).

Schlussfolgerungen: Eine gleichzeitige Behandlung mit einem SSRI und einem Statin scheint signifikante Vorteile hinsichtlich der antidepressiven Effektivität bei neutraler Auswirkung auf Suizidalität und Gesamtmortalität gegenüber einer alleinigen Behandlung mit einem SSRI zu haben.

\section{- Kommentar von Christian Lange-Asschenfeldt, Düsseldorf}

\section{Weitere Studien erscheinen gerechtfertigt}

Statine sind pleiotrope Substanzen, deren Wirksamkeit bei unterschiedlichen neuropsychiatrischen Störungen einschließlich neurodegenerativer und neuroinflammatorischer Erkrankungen diskutiert wird. Neben der Hemmung der HMGCoA-Reduktase als Schlüsselenzym der Cholesterinbiosynthese bestehen eine antioxidative und entzündungshemmende und immunmodulatorische Wirkung, zum Beispiel über eine zytokinvermittelte Modulation von T-Zellaktivierung und Expression antigenpräsentierender Zellen. Inflammatorische Prozesse mit erhöhter Aktivität insbesondere der Zytokine IL-6 und TNF-a sowie Erhöhung von C-reaktivem Protein und Kortisol wurden wiederholt bei der MDD nachgewiesen (z. B. [1]). Ein weiteres antidepressives therapeutisches Rationale ist ein antagonistischer Effekt am N-Methyl-D-Aspartat-(NMDA-) Rezeptor, wie tierexperimentell gezeigt wurde [2].

In bisherigen klinischen Studien konnten bereits antidepressive Augmentationseffekte von Simvastatin und Lovastatin nachgewiesen werden. Die jeweiligen Stichproben waren jedoch relativ klein und die Effekte beschränkten sich auf Response oder Symptomreduktion ohne Wirksamkeit hinsichtlich Remission. Die verbesserte Wirksamkeit einer SSRI-StatinKombinationsbehandlung auf die globalen Parameter der vorliegenden Studie sprechen grundsätzlich für eine klinische Relevanz. Zudem ergab sich für die Kombinationsbehandlung kein erhöhtes allgemeines Mortalitätsrisiko. Die Mortalität bei Kombinationsbehandlung spezifisch mit Lovastatin war je- doch erhöht. Eine erhöhte Suizidalität fand sich weder allgemein noch unter spezifischen SSRI-Statin-Kombinationsbehandlungen. Hinsichtlich spezifischer Substanzen war lediglich eine ausreichende statistische Power zur Beurteilung der Wirksamkeit für die Kombination Citalopram mit Simvastatin gegeben. Bei der Betrachtung der Ergebnisse der hier diskutierten Studie darf natürlich nicht vergessen werden, dass die Behandlungen nicht primär auf die Augmentation der antidepressiven Therapie abzielten, sondern dass es sich um Koinzidenzen handelte. Dennoch rechtfertigen die Ergebnisse sicher weitere Studien.
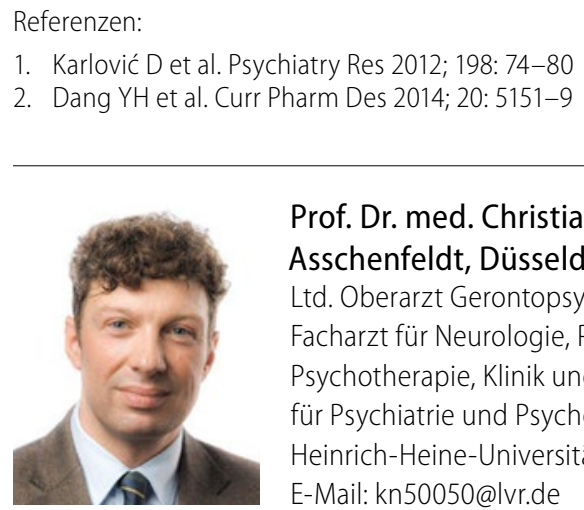

Prof. Dr. med. Christian Lange-

Asschenfeldt, Düsseldorf

Ltd. Oberarzt Gerontopsychiatrie,

Facharzt für Neurologie, Psychiatrie und

Psychotherapie, Klinik und Poliklinik

für Psychiatrie und Psychotherapie der

Heinrich-Heine-Universität, LVR-Klinikum

E-Mail:kn50050@|vr.de 